\title{
Ramanujan-type Congruences for Overpartitions Modulo 5
}

\author{
William Y.C. Chen ${ }^{a, b}$, Lisa H. Sun ${ }^{a, *}$, Rong-Hua Wang ${ }^{a}$ and Li Zhang ${ }^{a}$ \\ ${ }^{a}$ Center for Combinatorics, LPMC-TJKLC \\ Nankai University, Tianjin 300071, P. R. China \\ ${ }^{b}$ Center for Applied Mathematics \\ Tianjin University, Tianjin 300072, P. R. China \\ chen@nankai.edu.cn, *sunhui@nankai.edu.cn, \\ wangwang@mail.nankai.edu.cn, zhangli427@mail.nankai.edu.cn
}

\begin{abstract}
Let $\bar{p}(n)$ denote the number of overpartitions of $n$. Hirschhorn and Sellers showed that $\bar{p}(4 n+3) \equiv 0(\bmod 8)$ for $n \geq 0$. They also conjectured that $\bar{p}(40 n+$ $35) \equiv 0(\bmod 40)$ for $n \geq 0$. Chen and Xia proved this conjecture by using the $(p, k)$ parametrization of theta functions given by Alaca, Alaca and Williams. In this paper, we show that $\bar{p}(5 n) \equiv(-1)^{n} \bar{p}(4 \cdot 5 n)(\bmod 5)$ for $n \geq 0$ and $\bar{p}(n) \equiv(-1)^{n} \bar{p}(4 n)(\bmod 8)$ for $n \geq 0$ by using the relation of the generating function of $\bar{p}(5 n)$ modulo 5 found by Treneer and the 2-adic expansion of the generating function of $\bar{p}(n)$ due to Mahlburg. As a consequence, we deduce that $\bar{p}\left(4^{k}(40 n+35)\right) \equiv 0(\bmod 40)$ for $n, k \geq 0$. Furthermore, applying the Hecke operator on $\phi(q)^{3}$ and the fact that $\phi(q)^{3}$ is a Hecke eigenform, we obtain an infinite family of congrences $\bar{p}\left(4^{k} \cdot 5 \ell^{2} n\right) \equiv 0(\bmod 5)$, where $k \geq 0$ and $\ell$ is a prime such that $\ell \equiv 3(\bmod 5)$ and $\left(\frac{-n}{\ell}\right)=-1$. Moreover, we show that $\bar{p}\left(5^{2} n\right) \equiv \bar{p}\left(5^{4} n\right)$ $(\bmod 5)$ for $n \geq 0$. So we are led to the congruences $\bar{p}\left(4^{k} 5^{2 i+3}(5 n \pm 1)\right) \equiv 0(\bmod 5)$ for $n, k, i \geq 0$. In this way, we obtain various Ramanujan-type congruences for $\bar{p}(n)$ modulo 5 such as $\bar{p}(45(3 n+1)) \equiv 0(\bmod 5)$ and $\bar{p}(125(5 n \pm 1)) \equiv 0(\bmod 5)$ for $n \geq 0$.
\end{abstract}

Keywords: overpartition, Ramanujan-type congruence, modular form, Hecke operator, Hecke eigenform

MSC(2010): 05A17, 11P83

\section{Introduction}

The objective of this paper is to use half-integral weight modular forms to derive three infinite families of congruences for overpartitions modulo 5.

Recall that a partition of a nonnegative integer $n$ is a nonincreasing sequence of positive integers whose sum is $n$. An overpartition of $n$ is a partition of $n$ where the first occurrence of each distinct part may be overlined. We denote the number of overpartitions of $n$ by $\bar{p}(n)$. We set $\bar{p}(0)=1$ and $\bar{p}(n)=0$ if $n<0$. For example, there are eight overpartitions of 3

$$
3, \overline{3}, 2+1, \overline{2}+1,2+\overline{1}, \overline{2}+\overline{1}, 1+1+1, \overline{1}+1+1 .
$$


Overpartitions arise in combinatorics [6], q-series [5], symmetric functions [2], representation theory [11], mathematical physics [7, 8] and number theory [15, 16]. They are also called standard MacMahon diagrams, joint partitions, jagged partitions or dotted partitions.

Corteel and Lovejoy [6] showed that the generating function of $\bar{p}(n)$ is given by

$$
\sum_{n \geq 0} \bar{p}(n) q^{n}=\frac{(-q ; q)_{\infty}}{(q ; q)_{\infty}}
$$

Recall that the generating function of $\bar{p}(n)$ can be expressed as

$$
\sum_{n \geq 0} \bar{p}(n) q^{n}=\frac{1}{\phi(-q)}
$$

where $\phi(q)$ is Ramanujan's theta function as defined by

$$
\phi(q)=\sum_{n=-\infty}^{\infty} q^{n^{2}}
$$

see Berndt [2].

On the other hand, the generating function of $\bar{p}(n)$ has the following 2-adic expansion

$$
\sum_{n \geq 0} \bar{p}(n) q^{n}=1+\sum_{k=1}^{\infty} 2^{k} \sum_{n=1}^{\infty}(-1)^{n+k} c_{k}(n) q^{n}
$$

where $c_{k}(n)$ denotes the number of representations of $n$ as a sum of $k$ squares of positive integers. The above 2-adic expansion (1.2) is useful to derive congruences for $\bar{p}(n)$ modulo powers of 2, see, for example [12,13, 18].

By employing dissection formulas, Fortin, Jacob and Mathieu [7], Hirschhorn and Sellers [9] independently derived various Ramanujan-type congruences for $\bar{p}(n)$, such as

$$
\bar{p}(4 n+3) \equiv 0 \quad(\bmod 8)
$$

Hirschhorn and Sellers [9] proposed the following conjectures

$$
\begin{array}{ll}
\bar{p}(27 n+18) \equiv 0 & (\bmod 12), \\
\bar{p}(40 n+35) \equiv 0 & (\bmod 40) .
\end{array}
$$

They also conjectured that if $\ell$ is prime and $r$ is a quadratic nonresidue modulo $\ell$ then

$$
\bar{p}(\ell n+r) \equiv\left\{\begin{array}{llll}
0 & (\bmod 8) & \text { if } \ell \equiv \pm 1 \quad(\bmod 8) \\
0 & (\bmod 4) & \text { if } \ell \equiv \pm 3 & (\bmod 8)
\end{array}\right.
$$


By using the 3-dissection formula for $\phi(-q)$, Hirschhorn and Sellers [10] proved (1.4) and obtained a family of congruences

$$
\bar{p}\left(9^{\alpha}(27 n+18)\right) \equiv 0 \quad(\bmod 12),
$$

where $n, \alpha \geq 0$.

Employing the 2-dissection formulas of theta functions due to Ramanujan, Hirschhorn and Sellers [9], Chen and Xia [4] obtained a generating function of $\bar{p}(40 n+35)$ modulo 5 . Using the $(p, k)$-parametrization of theta functions given by Alaca, Alaca and Williams [1], they showed that

$$
\bar{p}(40 n+35) \equiv 0 \quad(\bmod 5) .
$$

This proves Hirschhorn and Sellers' conjecture (1.5) by combining congruence (1.3). Applying the 2-adic expansion (1.2), Kim [13] proved (1.6) and obtained congruence properties of $\bar{p}(n)$ modulo 8 .

For powers of 2, Mahlburg [18] showed that $\bar{p}(n) \equiv 0(\bmod 64)$ holds for a set of integers of arithmetic density 1 . Kim [12] showed that $\bar{p}(n) \equiv 0(\bmod 128)$ holds for a set of integers of arithmetic density 1. For the modulus 3, by using the fact that $\phi(q)^{5}$ is a Hecke eigenform in the half-integral weight modular form space $M_{\frac{5}{2}}\left(\tilde{\Gamma}_{0}(4)\right)$, Lovejoy and Osburn [17] proved that

$$
\bar{p}\left(3 \ell^{3} n\right) \equiv 0 \quad(\bmod 3)
$$

where $\ell \equiv 2(\bmod 3)$ is an odd prime and $\ell \nmid n$. Moreover, by utilizing half-integral weight modular forms, Treneer [21] showed that for a prime $\ell$ such that $\ell \equiv-1(\bmod 5)$,

$$
\bar{p}\left(5 \ell^{3} n\right) \equiv 0 \quad(\bmod 5)
$$

for all $n$ coprime to $\ell$.

In this paper, we establish the following two congruence relations for overpartitions modulo 5 and modulo 8 by using a relation of the generating function of $\bar{p}(5 n)$ modulo 5 and applying the 2-adic expansion (1.2).

Theorem 1.1. For $n \geq 0$, we have

$$
\bar{p}(5 n) \equiv(-1)^{n} \bar{p}(4 \cdot 5 n) \quad(\bmod 5) .
$$

Theorem 1.2. For $n \geq 0$, we have

$$
\bar{p}(n) \equiv(-1)^{n} \bar{p}(4 n) \quad(\bmod 8)
$$

Combining the above two congruence relations with congruences (1.3) and (1.7), we arrive at a family of congruences modulo 40 . 
Corollary 1.3. For $n, k \geq 0$, we have

$$
\bar{p}\left(4^{k}(40 n+35)\right) \equiv 0 \quad(\bmod 40) .
$$

Based on the Hecke operator on $\phi(q)^{3}$ and the fact that $\phi(q)^{3}$ is a Hecke eigenform in $M_{\frac{3}{2}}\left(\tilde{\Gamma}_{0}(4)\right)$, we obtain a family of congruences for overpartitions modulo 5 .

Theorem 1.4. Let $(\dot{\bar{l}})$ denote the Legendre symbol. Assume that $k$ is a nonnegative integer and $\ell$ is a prime with $\ell \equiv 3(\bmod 5)$. Then we have

$$
\bar{p}\left(4^{k} \cdot 5 \ell^{2} n\right) \equiv 0 \quad(\bmod 5),
$$

where $n$ is a nonnegative integer such that $\left(\frac{-n}{\ell}\right)=-1$.

Using the properties of the Hecke operator $T_{\frac{3}{2}, 16}\left(\ell^{2}\right)$ and the Hecke eigenform $\phi(q)^{3}$, we are led to another congruence relation for overpartitions modulo 5.

Theorem 1.5. For $n \geq 0$, we have

$$
\bar{p}\left(5^{2} n\right) \equiv \bar{p}\left(5^{4} n\right) \quad(\bmod 5) .
$$

Combining (1.8) and (1.11), we find the following family of congruences modulo 5 .

Corollary 1.6. For $n, k, i \geq 0$, we have

$$
\bar{p}\left(4^{k} 5^{2 i+3}(5 n \pm 1)\right) \equiv 0 \quad(\bmod 5) .
$$

\section{Preliminaries}

To make this paper self-contained, we recall some definitions and notation on half-integral weight modular forms. For more details, see [3, 14, 19 21].

Let $k$ be an odd positive integer and $N$ be a positive integer with $4 \mid N$. We use $M_{\frac{k}{2}}\left(\tilde{\Gamma}_{0}(N)\right)$ to denote the space of holomorphic modular forms on $\Gamma_{0}(N)$ of weight $\frac{k}{2}$.

Definition 2.1. Let

$$
f(z)=\sum_{n \geq 0} a(n) q^{n}
$$

be a modular form in $M_{\frac{k}{2}}\left(\tilde{\Gamma}_{0}(N)\right)$. For any odd prime $\ell \nmid N$, the action of the Hecke operator $T_{\frac{k}{2}, N}\left(\ell^{2}\right)$ on $f(z) \in M_{\frac{k}{2}}\left(\tilde{\Gamma}_{0}(N)\right)$ is given by

$$
f(z) \mid T_{\frac{k}{2}, N}\left(\ell^{2}\right)=\sum_{n \geq 0}\left(a\left(\ell^{2} n\right)+\left(\frac{(-1)^{\frac{k-1}{2} n}}{\ell}\right) \ell^{\frac{k-3}{2}} a(n)+\ell^{k-2} a\left(\frac{n}{\ell^{2}}\right)\right) q^{n},
$$

where $a\left(\frac{n}{\ell^{2}}\right)=0$ if $n$ is not divisible by $\ell^{2}$. 
The following proposition says that the Hecke operator $T_{\frac{k}{2}, N}\left(\ell^{2}\right)$ maps the modular form space $M_{\frac{k}{2}}\left(\tilde{\Gamma}_{0}(N)\right)$ into itself.

Proposition 2.2. Let $\ell$ be an odd prime and $f(z) \in M_{\frac{k}{2}}\left(\tilde{\Gamma}_{0}(N)\right)$, then

$$
f(z) \mid T_{\frac{k}{2}, N}\left(\ell^{2}\right) \in M_{\frac{k}{2}}\left(\tilde{\Gamma}_{0}(N)\right)
$$

A Hecke eigenform associated with the Hecke operator $T_{\frac{k}{2}, N}\left(\ell^{2}\right)$ is defined as follows.

Definition 2.3. A half-integral weight modular form $f(z) \in M_{\frac{k}{2}}\left(\tilde{\Gamma}_{0}(4 N)\right)$ is called a Hecke eigenform for the Hecke operator $T_{\frac{k}{2}, N}\left(\ell^{2}\right)$, if for every prime $\ell \nmid 4 N$ there exists a complex number $\lambda(\ell)$ for which

$$
f(z) \mid T_{\frac{k}{2}, N}\left(\ell^{2}\right)=\lambda(\ell) f(z)
$$

For the space of half-integral weight modular forms on $\Gamma_{0}(4)$, we have the following dimension formula.

Proposition 2.4. We have

$$
\operatorname{dim} M_{\frac{k}{2}}\left(\tilde{\Gamma}_{0}(4)\right)=1+\left\lfloor\frac{k}{4}\right\rfloor
$$

By the above dimension formula, we see that $\operatorname{dim} M_{\frac{3}{2}}\left(\tilde{\Gamma}_{0}(4)\right)=1$. From the fact that $\phi(q)^{3} \in M_{\frac{3}{2}}\left(\tilde{\Gamma}_{0}(4)\right)$, it is easy to deduce that

$$
\phi(q)^{3} \mid T_{\frac{3}{2}, 4}\left(\ell^{2}\right)=(\ell+1) \phi(q)^{3}
$$

see, for example [21, P. 18].

\section{Proofs of Theorem 1.1 and Theorem 1.2}

In this section, we give proofs of Theorem 1.1 and Theorem 1.2 by using a relation of the generating function of $\bar{p}(5 n)$ modulo 5 and the 2-adic expansion (1.2) of $\bar{p}(n)$.

Proof of Theorem 1.1. Recall the following 2-dissection formula for $\phi(q)$,

$$
\phi(q)=\phi\left(q^{4}\right)+2 q \psi\left(q^{8}\right)
$$

where

$$
\psi(q)=\sum_{n=0}^{\infty} q^{\frac{n^{2}+n}{2}}
$$


see, for example, Hirschhorn and Sellers [9]. Replacing $q$ by $-q$, (3.1) becomes

$$
\phi(-q)=\phi\left(q^{4}\right)-2 q \psi\left(q^{8}\right)
$$

We now consider the generating function of $\bar{p}(5 n)$ modulo 5 . The following relation is due to Treneer [21, p. 18],

$$
\sum_{n \geq 0} \bar{p}(5 n) q^{n} \equiv \phi(-q)^{3} \quad(\bmod 5)
$$

Plugging (3.2) into (3.3) yields that

$$
\sum_{n \geq 0} \bar{p}(5 n) q^{n} \equiv \phi\left(q^{4}\right)^{3}-q \phi\left(q^{4}\right)^{2} \psi\left(q^{8}\right)+2 q^{2} \phi\left(q^{4}\right) \psi\left(q^{8}\right)^{2}-3 q^{3} \psi\left(q^{8}\right)^{3} \quad(\bmod 5)
$$

Extracting the terms of $q^{4 n+i}$ for $i=0,1,2,3$ on both sides of (3.4) and setting $q^{4}$ to $q$, we obtain

$$
\begin{aligned}
& \sum_{n \geq 0} \bar{p}(20 n) q^{n} \equiv \phi(q)^{3} \quad(\bmod 5), \\
& \sum_{n \geq 0} \bar{p}(20 n+5) q^{n} \equiv-\phi(q)^{2} \psi\left(q^{2}\right) \quad(\bmod 5) \\
& \sum_{n \geq 0} \bar{p}(20 n+10) q^{n} \equiv 2 \phi(q) \psi\left(q^{2}\right)^{2} \quad(\bmod 5) \\
& \sum_{n \geq 0} \bar{p}(20 n+15) q^{n} \equiv-3 \psi\left(q^{2}\right)^{3} \quad(\bmod 5) .
\end{aligned}
$$

Substituting the 2-dissection formula (3.1) into (3.5), we find that

$$
\sum_{n \geq 0} \bar{p}(20 n) q^{n} \equiv \phi\left(q^{4}\right)^{3}+q \phi\left(q^{4}\right)^{2} \psi\left(q^{8}\right)+2 q^{2} \phi\left(q^{4}\right) \psi\left(q^{8}\right)^{2}+3 q^{3} \psi\left(q^{8}\right)^{3} \quad(\bmod 5)
$$

Extracting the terms of $q^{4 n+i}$ for $i=0,1,2,3$ on both sides of (3.9) and setting $q^{4}$ to $q$, we obtain

$$
\begin{aligned}
& \sum_{n \geq 0} \bar{p}(4 \cdot 20 n) \equiv \phi(q)^{3} \quad(\bmod 5), \\
& \sum_{n \geq 0} \bar{p}(4 \cdot(20 n+5)) \equiv \phi(q)^{2} \psi\left(q^{2}\right) \quad(\bmod 5), \\
& \sum_{n \geq 0} \bar{p}(4 \cdot(20 n+10)) \equiv 2 \phi(q) \psi\left(q^{2}\right)^{2} \quad(\bmod 5), \\
& \sum_{n \geq 0} \bar{p}(4 \cdot(20 n+15)) \equiv 3 \psi\left(q^{2}\right)^{3} \quad(\bmod 5) .
\end{aligned}
$$


Comparing the equations (3.5)-(3.8) with (3.10)-(3.13), we deduce that

$$
\begin{aligned}
& \bar{p}(5 \cdot(4 n)) \equiv \bar{p}(4 \cdot 5 \cdot 4 n) \quad(\bmod 5), \\
& \bar{p}(5 \cdot(4 n+1)) \equiv-\bar{p}(4 \cdot 5 \cdot(4 n+1)) \quad(\bmod 5), \\
& \bar{p}(5 \cdot(4 n+2)) \equiv \bar{p}(4 \cdot 5 \cdot(4 n+2)) \quad(\bmod 5), \\
& \bar{p}(5 \cdot(4 n+3)) \equiv-\bar{p}(4 \cdot 5 \cdot(4 n+3)) \quad(\bmod 5) .
\end{aligned}
$$

So we conclude that

$$
\bar{p}(5 n) \equiv(-1)^{n} \bar{p}(4 \cdot 5 n) \quad(\bmod 5) .
$$

This completes the proof.

We note that extracting the terms of odd powers of $q$ on both sides of (3.8) leads to the congruence $\bar{p}(40 n+35) \equiv 0(\bmod 5)$ due to Chen and Xia [4].

Next, we prove Theorem 1.2 by using the 2-adic expansion (1.2). Recall that $c_{k}(n)$ in (1.2) denotes the number of representations of $n$ as a sum of $k$ squares of positive integers. In particular, $c_{1}(n)=1$ if $n$ is a square; otherwise, $c_{1}(n)=0$.

Proof of Theorem 1.2. It follows from (1.2) that

$$
\bar{p}(n) \equiv(-1)^{n}\left(-2 c_{1}(n)+4 c_{2}(n)\right) \quad(\bmod 8),
$$

where $n \geq 1$. Replacing $n$ by $4 n$ in (3.14), we get

$$
\bar{p}(4 n) \equiv-2 c_{1}(4 n)+4 c_{2}(4 n) \quad(\bmod 8) .
$$

Since $c_{1}(n)=c_{1}(4 n)$ and $c_{2}(n)=c_{2}(4 n)$, (3.15) can be rewritten as

$$
\bar{p}(4 n) \equiv-2 c_{1}(n)+4 c_{2}(n) \quad(\bmod 8) .
$$

Substituting (3.16) into (3.14), we arrive at

$$
\bar{p}(n) \equiv(-1)^{n} \bar{p}(4 n) \quad(\bmod 8),
$$

as claimed.

It is easy to see that Corollary 1.3 can be obtained by iteratively applying Theorem 1.1 and Theorem 1.2 to the congruences $\bar{p}(40 n+35) \equiv 0(\bmod 5)$ and $\bar{p}(40 n+35) \equiv 0$ (mod 8) that can be deduced from congruence (1.3) by replacing $n$ with $10 n+8$.

\section{Proof of Theorem 1.4}

In this section, we prove Theorem 1.4 by using the Hecke operator on $\phi(q)^{3}$ along with the fact that $\phi(q)^{3}$ is a Hecke eigenform in $M_{\frac{3}{2}}\left(\tilde{\Gamma}_{0}(4)\right)$.

In view of Theorem 1.1, to prove Theorem 1.4, it suffices to consider the special case $k=0$ that takes the following form. 
Theorem 4.1. Let $\ell$ be a prime with $\ell \equiv 3(\bmod 5)$. Then

$$
\bar{p}\left(5 \ell^{2} n\right) \equiv 0 \quad(\bmod 5)
$$

holds for any nonnegative integer $n$ with $\left(\frac{-n}{\ell}\right)=-1$.

Proof. Recall that $\phi(-q)^{3}$ is a modular form in $M_{\frac{3}{2}}\left(\tilde{\Gamma}_{0}(16)\right)$. Suppose that

$$
\phi(-q)^{3}=\sum_{n \geq 0} a(n) q^{n}
$$

is the Fourier expansion of $\phi(-q)^{3}$.

Applying the Hecke operator $T_{\frac{3}{2}, 16}\left(\ell^{2}\right)$ to $\phi(-q)^{3}$ and using (2.1), we find that

$$
\phi(-q)^{3} \mid T_{\frac{3}{2}, 16}\left(\ell^{2}\right)=\sum_{n=0}^{\infty}\left(a\left(\ell^{2} n\right)+\left(\frac{-n}{\ell}\right) a(n)+\ell a\left(\frac{n}{\ell^{2}}\right)\right) q^{n},
$$

where $\ell$ is an odd prime. Replacing $q$ by $-q$ in (2.2), we see that $\phi(-q)^{3}$ is a Hecke eigenform in the space $M_{\frac{3}{2}}\left(\tilde{\Gamma}_{0}(16)\right)$, and hence

$$
\phi(-q)^{3} \mid T_{\frac{3}{2}, 16}\left(\ell^{2}\right)=(\ell+1) \phi(-q)^{3} .
$$

Comparing the coefficients of $q^{n}$ in (4.3) and (4.4), we deduce that

$$
a\left(\ell^{2} n\right)+\left(\frac{-n}{\ell}\right) a(n)+\ell a\left(\frac{n}{\ell^{2}}\right)=(\ell+1) a(n) .
$$

Revoking the congruence (3.3), that is,

$$
\phi(-q)^{3} \equiv \sum_{n \geq 0} \bar{p}(5 n) q^{n} \quad(\bmod 5),
$$

and comparing (4.2) with (4.6), we get

$$
a(n) \equiv \bar{p}(5 n) \quad(\bmod 5) .
$$

Plugging (4.7) into (4.5), we deduce that

$$
\bar{p}\left(5 \ell^{2} n\right)+\left(\frac{-n}{\ell}\right) \bar{p}(5 n)+\ell \bar{p}\left(\frac{5 n}{\ell^{2}}\right) \equiv(\ell+1) \bar{p}(5 n) \quad(\bmod 5) .
$$

Since $\ell \equiv 3(\bmod 5)$ and $\left(\frac{-n}{\ell}\right)=-1$, we see that $\ell \nmid 5$ and $\ell \nmid n$, so that $\ell^{2} \nmid 5 n$ and $\bar{p}\left(\frac{5 n}{\ell^{2}}\right)=0$. Moreover, we have $\left(\frac{-n}{\ell}\right) \equiv(\ell+1) \equiv-1(\bmod 5)$. Hence congruence (4.8) becomes

$$
\bar{p}\left(5 \ell^{2} n\right) \equiv 0 \quad(\bmod 5) .
$$

This completes the proof. 
We now give some special cases of Theorem 1.4. Setting $\ell=3$ and $k=0,1$ in Theorem 1.4, respectively, we obtain the following congruences for $n \geq 0$,

$$
\begin{aligned}
& \bar{p}(45(3 n+1)) \equiv 0 \quad(\bmod 5), \\
& \bar{p}(180(3 n+1)) \equiv 0 \quad(\bmod 5)
\end{aligned}
$$

Setting $\ell=13, k=0$ in Theorem 1.4, we obtain the following congruences for $n \geq 0$,

$$
\begin{aligned}
& \bar{p}(845(13 n+2)) \equiv 0 \quad(\bmod 5) \\
& \bar{p}(845(13 n+5)) \equiv 0 \quad(\bmod 5) \\
& \bar{p}(845(13 n+6)) \equiv 0 \quad(\bmod 5) \\
& \bar{p}(845(13 n+7)) \equiv 0 \quad(\bmod 5) \\
& \bar{p}(845(13 n+8)) \equiv 0 \quad(\bmod 5) \\
& \bar{p}(845(13 n+11)) \equiv 0 \quad(\bmod 5)
\end{aligned}
$$

\section{Proof of Theorem 1.5}

In this section, we complete the proof of Theorem 1.5 by using the Hecke operator $T_{\frac{3}{2}, 16}\left(\ell^{2}\right)$ and the Hecke eigenform $\phi(-q)^{3}$.

Proof of Theorem 1.5. Setting $\ell=5$ in the congruence relation (4.8), we find that

$$
\bar{p}(5 n) \equiv \bar{p}\left(5^{3} n\right)+\left(\frac{n}{5}\right) \bar{p}(5 n) \quad(\bmod 5) .
$$

By the definition of the Legendre symbol, we see that if $n \equiv 0(\bmod 5)$, then $\left(\frac{n}{5}\right)=0$. Hence, by replacing $n$ with $5 n$ in congruence (5.1), we obtain that

$$
\bar{p}\left(5^{2} n\right) \equiv \bar{p}\left(5^{4} n\right) \quad(\bmod 5)
$$

as claimed.

Furthermore, we note that if $n \equiv \pm 1(\bmod 5)$, then $\left(\frac{n}{5}\right)=1$. Hence by setting $n$ to $5 n \pm 1$ in (5.1), we deduce that

$$
\bar{p}\left(5^{3}(5 n \pm 1)\right) \equiv 0 \quad(\bmod 5) .
$$

By iteratively applying the congruence $\bar{p}(5 n) \equiv(-1)^{n} \bar{p}(4 \cdot 5 n)(\bmod 5)$ given in Theorem 1.1 and congruence (5.2) to (5.3), we obtain that

$$
\bar{p}\left(4^{k} 5^{2 i+3}(5 n \pm 1)\right) \equiv 0 \quad(\bmod 5)
$$


where $n, k, i \geq 0$. This proves Corollary 1.6.

For $n \geq 0$, setting $i=0$ and $k=0,1$ in (5.4), we obtain the following special cases

$$
\begin{aligned}
& \bar{p}(125(5 n \pm 1)) \equiv 0 \quad(\bmod 5) \\
& \bar{p}(500(5 n \pm 1)) \equiv 0 \quad(\bmod 5) .
\end{aligned}
$$

By replacing $n$ by $5 n \pm 2$ in (5.1) and iteratively using the congruence relation (5.2), we obtain the following relation.

Corollary 5.1. For $n, i \geq 0$, we have

$$
\bar{p}(5(5 n \pm 2)) \equiv 3 \bar{p}\left(5^{2 i+3}(5 n \pm 2)\right) \quad(\bmod 5) .
$$

Acknowledgments. This work was supported by the 973 Project, the PCSIRT Project of the Ministry of Education and the National Science Foundation of China.

\section{References}

[1] A. Alaca, S. Alaca and K.S. Williams, On the two-dimensional theta functions of the Borweins, Acta Arith. 124 (2006) 177-195.

[2] B.C. Berndt, Number Theory in the Spirit of Ramanujan, American Mathematical Society, Providence, RI, 2006.

[3] W.Y.C. Chen, D.K. Du, Q.H. Hou and L.H. Sun, Congruences of multipartition functions modulo powers of primes, Ramanujan J., to appear.

[4] W.Y.C. Chen and E.X.W. Xia, Proof of a conjecture of Hirschhorn and Sellers on overpartitions, Acta Arith. 163 (1) (2014) 59-69.

[5] S. Corteel and P. Hitczenko, Multiplicity and number of parts in overpartitions, Ann. Combin. 8 (2004) 287-301.

[6] S. Corteel and J. Lovejoy, Overpartitions, Trans. Amer. Math. Soc. 356 (2004) 16231635.

[7] J.-F. Fortin, P. Jacob and P. Mathieu, Jagged partitions, Ramanujan J. 10 (2005) $215-235$.

[8] J.-F. Fortin, P. Jacob and P. Mathieu, Generating function for $K$-restricted jagged partitions, Electron. J. Combin. 12 (1) (2005) R12.

[9] M.D. Hirschhorn and J.A. Sellers, Arithmetic relations for overpartitions, J. Combin. Math. Combin. Comput. 53 (2005) 65-73. 
[10] M.D. Hirschhorn and J.A. Sellers, An infinite family of overpartition congruences modulo 12, Integers 5 (2005) \#A20.

[11] S.-J. Kang and J.-H. Kwon, Crystal bases of the fock space representations and string functions, J. Algebra 280 (2004) 313-349.

[12] B. Kim, The overpartition function modulo 128, Integers 8 (2008) \#A38.

[13] B. Kim, A short note on the overpartition function, Discrete Math. 309 (2009) 25282532.

[14] N. Koblitz, Introduction to Elliptic Curves and Modular Forms, Springer, New York, 1993.

[15] J. Lovejoy, Overpartitions and real quadratic fields, J. Number Theory 106 (2004) 178-186.

[16] J. Lovejoy and O. Mallet, Overpartition pairs and two classes of basic hypergeomtric series, Adv. Math. 217 (2008) 386-418.

[17] J. Lovejoy and R. Osburn, Quadratic forms and four partition functions modulo 3, Integers 11 (2011) \#A4.

[18] K. Mahlburg, The overpartition function modulo small powers of 2, Discrete Math. 286 (2004) 263-267.

[19] K. Ono, The Web of Modularity: Arithmetic of the Coefficients of Modular Forms and $q$-Series, CBMS Regional Conference Series in Mathematics 102, AMS Press, Providence, RI, 2004.

[20] G. Shimura, On modular forms of half-integral weight, Ann. Math. 97 (1973) 440481.

[21] S. Treneer, Congruences for the coefficients of weakly holomorphic modular forms, Proc. London Math. Soc. 93 (2006) 304-324. 